
[0212-7199(2002) 19: 9; pp 466-469]
ANALES DE MEDICINA INTERNA
Copyright @ 2002 ARAN EDICIONES, S.L.

An. Med. InTERna (Madrid) Vol. 19, N. $^{\circ} 9$, pp. $466-469,2002$

\title{
Pseudoartrosis vertebral en la espondilitis anquilosante
}

\author{
F. J. MANERO RUIZ, M. ROCA ESPIAU*, J. MOTA MARTÍNEZ* \\ Servicios de Reumatología y *Radiología. Hospital Universitario Miguel Servet. \\ Zaragoza
}

SPINAL PSEUDOARTHROSIS IN ANKYLOSING SPONDYLITIS

\begin{abstract}
RESUMEN
La pseudoartrosis vertebral es una espondilodiscitis aséptica que aparece como complicación tardía de la espondilitis anquilosante. Dos pacientes presentaron este proceso de destrucción discovertebral después de una caída espontánea. Los dos asociaron manifestaciones neurológicas por compresión medular, interviniéndose quirúrgicamente el primero. En el estudio de estas lesiones, las diferentes técnicas de imagen se complementan. Con radiología simple se puede identificar en algunos casos la afección discovertebral. La tomografía axial computerizada delimita mejor estas lesiones y revela la fractura del arco posterior. Por último, el estudio con resonancia magnética aporta sensibilidad para la detección precoz, descarta la existencia de partes blandas paravertebrales que puedan ser de otro origen e identifica la compresión del cordón medular.
\end{abstract}

PALABRAS CLAVE: Pseudoartrosis vertebral. Espondilodiscitis. Espondilitis anquilosante. Resonancia magnética.

\begin{abstract}
Spinal pseudoarthrosis is an uncommon complication in patients with advanced ankylosing spondylitis which consists in destruction of the discovertebral junction. Two cases of spinal pseudoarthrosis at the tho racolumbar level after a spontaneous fall are reported. Because of the neural arch involvement and the spinal cord compression a stabilization was required. Different imaging techniques are complementaries in the study of this entity, conventional radiographs and computed tomography may depicte bone abnormalities, but magnetic resonance allows a correct evaluation of spinal cord and soft tissue involvement.
\end{abstract}

KEY WORDS: Spinal pseudoarthrosis. Spondylodiscitis. Ankylosing spondylitis. Magnetic resonance.

Manero Ruiz FJ, Roca Espiau M, Mota Martinez J. Pseudoartrosis vertebral en la espondilitis anquilosante. An Med Interna (Madrid) 2002; 19: 466-469.

\section{INTRODUCCIÓN}

La espondilitis anquilosante (EA) es un proceso inflamatorio preferentemente axial, que se caracteriza por entesopatía y artritis (1). La espondilodiscitis (aséptica) es una complicación poco frecuente de la EA que puede aparecer en cualquier momento de su evolución. Cawley (2) describe tres tipos diferentes de espondilodiscitis: a) forma periférica, que afecta a la zona periférica de la unión discovertebral; b) forma central, que se localiza en la parte central cartilaginosa de la unión discovertebral; y c) forma central y periférica, cuando la lesión destructiva se extiende a toda la superficie discal. Las formas 1 y 2 suelen encontrarse en fases iniciales de la EA, mientras que la forma 3 lo hace en fases tardías. Esta última lesión, llamada habitualmente pseudoartrosis vertebral (PsV) (3), ocurre en columnas vertebrales anquilosadas y osteoporóticas, que son mas frágiles ante un traumatismo. Describimos dos pacientes con esta complicación que asociaron compresión neurológica.

\section{CASOS APORTADOS}

Caso 1: Varón de 45 años diagnosticado de EA B27 positiva de 15 años de evolución. La columna dorsal y lumbar están totalmente limitadas con anquilosis de sacroilíacas y articulaciones interapofisarias dorsales y lumbares, calcificación discal y del ligamento interespinoso. Hace 5 meses sufre una caída con traumatismo vertebral, y aparición de dolor brusco en la unión dorsolumbar, que de forma progresiva asocia dolor en EEII con trastornos de la marcha que le impiden la deambulación, disestesias en pies, impotencia sexual, incontinencia de esfínteres. En la exploración clínica hay anestesia en silla de montar, abolición del reflejo cremastérico

Trabajo aceptado: 3 de abril de 2001

Correspondencia: Fco. Javier Manero Ruiz. Corona de Aragón 18-20, $3^{\circ}$ A. 50009 Zaragoza. 
derecho, abolición de los reflejos rotulianos y aquíleos, el babinsky es negativo y existe debilidad de la flexión en muslos, piernas y mas intenso en pies. Se encuentra afebril, sin adenopatías ni visceromegalias. Analíticamente: VSG: $7 \mathrm{~mm} 1^{\mathrm{a}}$ hora; función hepatorenal, hemograma, VIH, serología de brucella, salmonella, cultivos para gérmenes piógenos y micobacterias son normales o negativos. Mantoux: $10 \mathrm{~mm}$ a las 24 horas. En la radiografía: segmento D12L1 con destrucción discal, osteolisis y esclerosis de las vértebras adyacentes. La TAC dorsolumbar en D12-L1 (Fig. 1): osteolisis vertebral, destrucción discal con "fenómeno de vacío intradiscal" y fractura del arco posterior. La radiografía de tórax es normal. Se practicó gammagrafía con 99Tc MDP con captación del isótopo en D12-L1. En la RM (Fig. 2) con secuencias SE T1, T2 y T1 gadolinio: reabsorción importante del hueso esponjoso y subcondral en los platillos D12 y L1. La lesión condiciona espondilolistesis con afectación epidural y efecto compresivo medular, provocado por el arco posterior y las articulaciones interapofisarias. Ante la posibilidad de una discitis tuberculosa, se realizó punción del disco D12L1 y se inició tratamiento con tuberculostáticos, que son suspendidos al establecer la sospecha de pseudoartrosis. Una segunda gammagrafía con Galio 67 fue normal. La punción del disco D12L1 era estéril, sin granulomas y todos los estudios bacteriológicos fueron negativos. El paciente en ese momento tiene lesiones neurológicas irreversibles, precisa silla de ruedas y muletas para desplazarse. Además persiste el dolor a la flexión en la unión dorsolumbar por lo que se realiza intervención quirúrgica con fijación del segmento D12-L1.

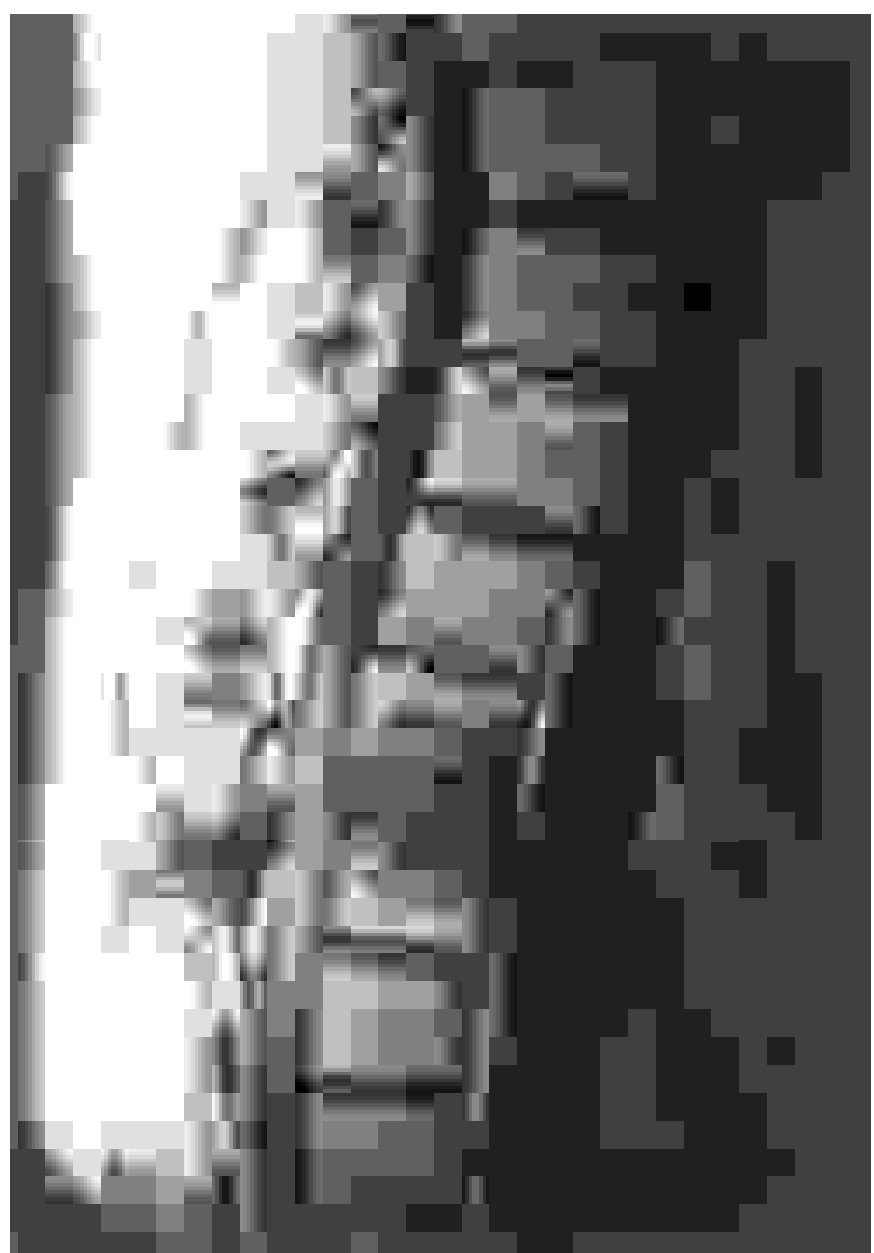

Fig. 1. Caso 1. TAC con reconstrucción sagital: línea de fractura sobre arco posterior. Esclerosis y vacio intradiscal.

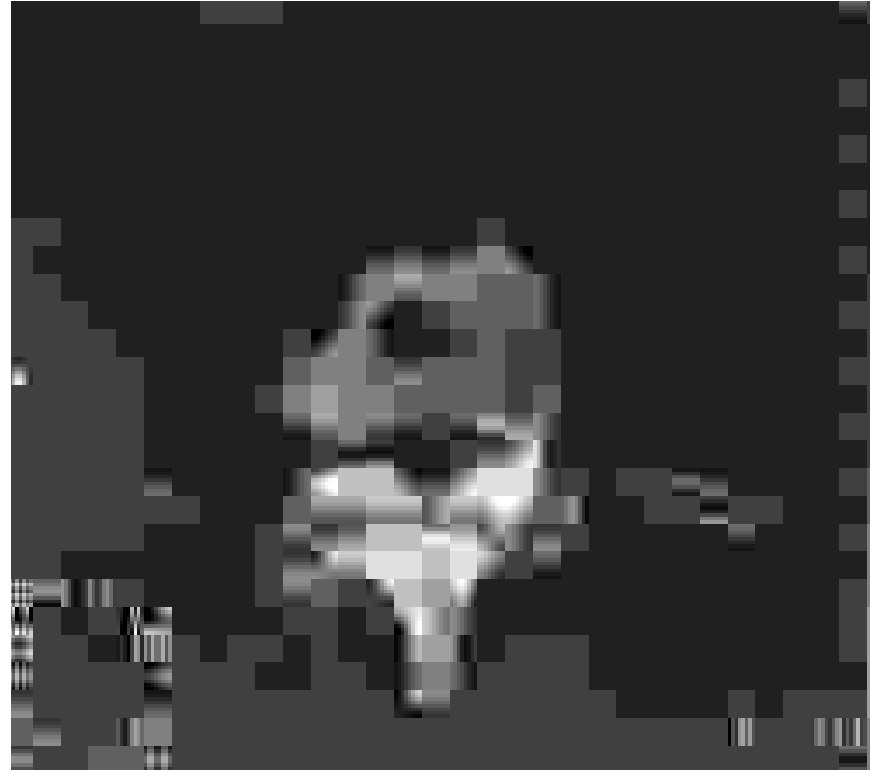

Fig. 2. Caso 1. RM : SE-T1: Destrucción discovertebral D12-L1 con hiposeñal. Espondilolístesis y reducción del diámetro del canal lumbar, condicionado por las lesiones del arco posterior que desplazan el cordón medular.

Caso 2: Varón de 47 años con antecedentes de etilismo y EA B27 positiva desde hace 20 años. La columna vertebral está limitada totalmente en los tres niveles: cervical, dorsal y lumbar con radiografía de anquilosis sacroilíaca, sindesmofitosis y artritis interapofisaria. No tiene artritis periférica, psoriasis o enfermedad inflamatoria intestinal y utiliza AINE de forma constante. A raíz de una caída hace 2 años aparece bruscamente dolor con fractura de arco posterior D11-D12 y progresiva destrucción discovertebral. Hace 6 meses nueva caída que incrementa el dolor vertebral e inicia debilidad progresiva en EEII y alteración de los esfínteres. Sangre: VSG: $20 \mathrm{~mm}$, fosfatasa alcalina $261 \mathrm{UI} / \mathrm{L}$ (33-230), albúmina: $45,9 \mathrm{~g} / \mathrm{dl}(54,7-68,7)$, gammaglobulinas $20,8 \%$, GOT $54 \mathrm{UI} / \mathrm{L}$ (540), leucocitos 4.400, hemoglobina 13,7 g/dl, VCM 121, HCM 49,6 , plaquetas 93.000. Gammagrafía ósea con 99MDP TC hay captación en D11-D12, L2. Radiografía dorsal y lumbar con destrucción discal y osteolisis vertebral en el segmento D11-D12. TAC: D11- D12 con línea de fractura sobre un arco posterior esclerótico. RM con importante reabsorción discovertebral D11-D12, hipointenso en T1 e hiperintenso en T2, y abombamiento del muro posterior. Existe hipertrofia del arco posterior secundaria a la fractura que unido al abombamiento del muro posterior, provoca compresión medular manifestada por engrosamiento, pérdida de espacios subaracnoideos y aumento de la señal del cordon medular en T2. La medular intraesponjosa de L2 presenta edema hipointenso en $\mathrm{T} 1$ e hiperintenso en $\mathrm{T} 2$ correspondiente a un aplastamiento postraumático. El paciente utilizaba faja ortopédica y declinó la intervención quirúrgica para descompresión medular en el segmento D11-D12.

\section{DISCUSIÓN}

En nuestros dos pacientes con una EA de larga evolución, existe una relación directa entre el traumatismo, la fractura del arco posterior y la aparición de PsV. Un traumatismo puede desestabilizar un segmento vertebral alterado previamente, o bien determinar una fractura del arco poste- 
rior con un segmento vertebral inestable. La fractura del arco posterior como desencadenante es reconocida por algunos autores $(4,5)$ pero no aparece en todos los casos $(6,7)$. Se han descrito tres posibles causas de formación de PsV (3): a) un segmento no fusionado en una columna anquilosada que sufre hipermovilidad; b) una fractura aguda en un segmento ya fusionado; y c) una columna cifótica que sufre stress preferentemente en la unión dorsolumbar. Hay controversia en relación a los mecanismos que producen la destrucción vertebral. Para explicarlo existe una hipótesis mecánica, donde lo primero es el proceso degenerativo y la inflamación es secundaria $(2,8-10)$ y otra hipótesis inflamatoria donde los trastornos degenerativos serían secundarios al proceso inflamatorio inicial $(7,11)$. La histología, en los casos que se ha realizado, tiene pocos cambios inflamatorios predominando la hemorragia, el tejido fibroso y el callo óseo, por lo que puede ser más un contexto traumático que inflamatorio $(1,12)$. Se ha sugerido que en el momento de hacer las biopsias ya es tarde para diferenciar el origen mecánico o inflamatorio, por lo que los estudios histológicos deberían realizarse lo antes posible (13).

La PsV es una complicación tardía que aparece en EA de más de 10 años de evolución (6), con anquilosis vertebral y donde los pacientes ya se encuentran asintomáticos de su enfermedad (12). La PsV puede descubrirse ocasionalmente $(3,14)$ o ser sintomática, con dolor mecánico en el disco afectado que mejora con el reposo $(2,4)$. El desencadenante traumático puede ser mínimo y pasar desapercibido (5), pero es el punto de partida para el dolor, que puede ser abrupto como ocurrió en nuestros casos (12). Esta complicación no es tan infrecuente como inicialmente se ha pensado, ya que sólo se suelen detectar los casos sintomáticos. La frecuencia de formas sintomáticas en la EA es aproximadamente del 1,4\% (7). En un estudio reciente de 147 EA se encuentran un $8 \%$ (12 casos) de PsV de las que sólo el $17 \%$ ( 2 casos) eran sintomáticas (6). Raramente descrita en la región cervical (15), la localización preferente es la unión toracolumbar (2) y generalmente en un sólo nivel, provocando en ocasiones lesiones neurológicas que requerirán cirugia descompresiva (9).

La radiología sirve para confirmar la existencia de EA y detecta la destrucción del disco y platillos vertebrales adyacentes con esclerosis. La tomografía axial computerizada (TAC) es superior a la radiología en el estudio de las lesiones óseas. Localiza claramente la osteolisis vertebral, el "fenómeno de vacio intradiscal", descarta la existencia de "hinchazón de partes blandas paravertebrales" y sobre todo identifica la fractura del arco posterior (16), como ocurría en la reconstrucción sagital de nuestro primer caso (Fig. 1). La resonancia magnética $(\mathrm{RM})$ destaca por su gran sensibilidad para la detección de PsV que no es superada por otras técnicas no invasivas (17), además es la única prueba que muestra compresión medular condicionada por el desplazamiento de cuerpos vertebrales, provocado por la fractura del arco posterior. En los dos casos la RM detectó las alteraciones de señal en médula ósea correspondientes a edema y las de hueso subcondral en forma de reabsorción (hipointensidad en T1), descartando la afectación de partes blandas paravertebrales visibles en procesos infecciosos (Fig. 2). También delimita el tejido de granulación cicatricial, que presenta moderada captación de gadolinio y que contribuye a la reducción del diámetro del canal vertebral con secundario efecto compresivo. La RM identifica en el segundo paciente una fractura del cuerpo vertebral de L2, que no se veía en la radiografía simple, e indica la vulnerabilidad de esta columna vertebral ante los traumatismos.

La destrucción discovertebral que provoca la PsV plantea diagnóstico diferencial con los procesos infecciosos. El estudio con la gammagrafía ósea que combina tecnecio y galio es un método para sugerir el origen infeccioso cuando captan los dos isótopos (18). La ausencia de masa de partes blandas en la RM es otra diferencia importante con las infecciones (12). Además, esta técnica pone en evidencia en la PsV la falta de incremento de señal en el disco y los tejidos paravertebrales (5), aunque no es específico de las espondilitis infecciosas, debido al similar componente destructivo en los platillos vertebrales causado por la inestabilidad.

El tratamiento es conservador si no hay déficit neurológico ni fractura del arco posterior. Si el dolor es continuo y la seudoartrosis o subluxación progresa se realiza fijación. Cuando hay trastornos neurológicos se practica una descompresión (19). La radiología y la TAC permiten seguir la evolución, y cuando se interviene quirúrgicamante valorar la curación o refractura (20). Concluimos que el diagnóstico precoz de la PsV puede resultar difícil. En los dos pacientes presentados este diagnóstico se retrasó varios meses, cuando las lesiones eran evidentes. Por ello, el contexto clínico y sobre todo el estudio por imagen con una valoración cuidadosa de la RM, deberían ser suficientes para evitar otras exploraciones o realizar tratamientos que no correspondan.

\section{Bibliografía}

1. Calin A, Robertson D. Spondylodiscitis and pseudoarthrosis in a patient with enteropathic spondyloarthropathy. Ann Rheum Dis 1991; 50: 117 -

2. Cawley MID, Chalmers TM, Kellgren JH, Ball J. Destructive lesions of vertebral bodies in ankylosing spondylitis. Ann Rheum Dis 1972; 31: 345-58.

3. Fang D, Leong JCY, Ho EKW, Chan FL, Chow SP. Spinal pseudoarthrosis in ankylosing spondylitis. J Bone Joint Surg (Br). 1988; 70-B: 443-7.
4. Martel W. Spinal pseudoarthrosis. A complication of ankylosing spondylitis. Arthritis Rheum 1978; 21: 485-90.

5. Petterson T, Laasonen L, Leirisalo-Repo M, Tervahartiala P. Spinal pseudoarthrosis complicating ankylosingspondylitis: A report of two patients. Br J Rheumatol 1996; 34: 1319-23.

6. Kabasakal Y, Garrett SL, Calin A. The epidemiology of spondylodiscitis in ankylosing spondylitis-A controlled study. Br J Rheumatol 1996; 35: 660-3

7. Rasker JJ, Prevo RL, Lanting PJH. Spondylodiscitis in ankylosiing 
spondylitis, inflammation or trauma? Adescription of six cases. Scand J Rheumatol. 1996; 25: 52-7.

8. Cawley MID, Chalmers TM, Ball J. Destructive lesions of vertebral bodies in ankylosing spondylitis. Ann Rheum Dis 1971; 30: 539-40.

9. Hunter T, Dubo HIC. Spinal fractures complicating ankylosing spondylitis. A long-term followup study. Arthritis Rheum 1983; 26: 751-9.

10. Jajic I, Furst Z, Vuksic B. Spondylitis erosiva: report on 9 patients. Ann Rheum Dis 1982; 41: 237-41.

11. Sutherland RIL, Matheson D. Mecanisms of vertebral destruction in ankylosing spondylitis. Arthritis Rheum 1974; 17: 502.

12. Resnick D, Niwayama G. Ankylosing spondylitis. In: Resnick D, Niwayama $G$ (eds.): Diagnosis of bone and joint disorders. Philadelphia: Saunders Company. 1988; 1103-70.

13. Courtois C, Fallet GH, Vischer TL, Wettstein P. Erosive spondylopathy. Ann Rheum Dis 1980; 39: 462-8.

14. Little H, Urowitz MB, Smythe HA, Rosen PS. Asymptomatic spondylo- discitis. An unusual feature of ankylosing spondylitis. Arthritis Rheum 1974; 17: 487-93.

15. Lim KL, Chaudhuri K, Johnston RA, Sturrock RD. Cervical Spondylodiscitis in a patient with ankylosing spondylitis. Br J Rheumatol 1996; 35: $1037-8$.

16. Chan FL, Ho EKW, Chau EMT. Spinal pseudoarthrosis complicating spondylitis: comparison of CT and conventional tomography. Am J Roentgenol 1988; 150: 611-4.

17. Goldberg AL, Keaton NL, Rothfus WE, Daffner RH. Anquilosing spondylitis complicated by trauma: MR findings correlates with plain radiographs and CT. Skeletal Radiol 1993; 22: 333-6.

18. McCune WJ. Monoarticular arthritis. In: Kelley WN, Harris DE, Ruddy S y Sledge SD (Eds): Textbook of Rheumatology. Philadelphia: Saunders Company. 1989; 442-54.

19. Fox MW, Onofrio BM, Kilgore JE. Neurological complications of ankylosing spondylitis. J Neurosurg 1993; 78: 871-8.

20. Peh WCG, Luck KDK. Pseudoarthrosis in ankylosing spondylitis. Ann Rheum Dis 1994; 53: 206-10. 